\title{
Fuzzy Control of Rotor System Using an Electromagnetic Actuator
}

\author{
E. H. Koroishi ${ }^{1}$, V. Steffen $\mathrm{Jr}^{1}$ and J. Mahfoud ${ }^{2}$ \\ ${ }^{1}$ Federal University of Uberlândia (UFU) - School of Mechanical Engineering - Campus Santa Mônica, Av. João \\ Naves de Ávila 2121, Bloco 1M, Uberlândia, MG, Brazil, ZIP CODE 38400-902 \\ ${ }^{2}$ Lyon University, LAMCOS, INSA de Lyon, 20, Av. Albert Einstein, 69629 Villeurbanne, France
}

\begin{abstract}
In recent years, a number of new methods dedicated to acoustic and vibration attenuation have been developed and proposed aiming at handling several types of engineering problems related to the dynamic behavior of the system. This is mainly due to the demand for better performance and safer operation of mechanical systems. There are various types of actuators available. The present contribution is dedicated to the electromagnetic actuator (EMA). EMA uses electromagnetic forces to support the rotor without mechanical contact. Due to the size of the system model, it was necessary to reduce the model of the rotating system. EMA is represented by a nonlinear model, which justifies the use of Fuzzy Logic Control. The results obtained with nonlinear model are compared with the linear model of the actuator. In the linear case, the controllers are designed for two situations, namely $H_{\infty}$ norm and LQR, solved by LMIs in both cases. These techniques are able to take into account uncertainties in the system. In the control, the nonlinear electrodynamics forces of the actuators are considered as containing uncertainties. Simulation results demonstrate the effectiveness of the methodology conveyed.
\end{abstract}

\section{Introduction}

Currently, an increase of research works in engineering dedicated to the development of active vibration control techniques (AVC) is observed. This effort is stimulated by the necessity of lighter structures associated to higher operational performance and smaller operating costs [1]. In the last decades, the methodologies of AVC have received significant contributions, due particularly to the advances in the digital processing of signals and new methodologies of control as can be seen in [2-5]. Some of these contributions have caused deep impact in aerospace and robotic applications [6].

In terms of rotating machines, an important AVC methodology uses Active Magnetic Bearings. The AMB is a feedback mechanism that supports a spinning shaft by levitating it in a magnetic field [7]. For its operation, the sensor measures the relative position of the shaft and the measured signal is sent to the controller where it is processed. Then, the signal is amplified and fed as electric current into the coils of the magnet, generating an electromagnetic field that keeps the shaft in a desired position. The strength of the magnetic field depends both on the air gap between the shaft and the magnet and the dynamics of the system including the design of the controller.

In this work, the electromagnetic actuator is used to control the flexible rotor. However, in this type of actuator a nonlinear behaviour is found. Some authors propose to use an inverse model of the actuator to overcome this difficulty. They use the feedback technique to determine the control force and this force is used to determine the electrical current that drives the electromagnetic actuator.

In the present contribution, two techniques are compared. The first considers the nonlinear actuator in which the control strategy is based on a fuzzy controller of Takagi-Sugeno type. It was found to be well adapted for controlling flexible structures. Moreover, this type of controller allows taking into account slight nonlinearities and uncertainties due to the experimental context [9]. The second one considers the linear approach for the electromagnetic actuator. The controller is obtained by using the $H_{\infty}$ norm and Linear Quadratic Regulator (LQR); both methods were solved by Linear Matrix Inequalities (LMIs). In the following the EMA is first presented, and then the studied rotor model is shown before giving details regarding the control approaches used. Finally, numerical simulations are performed and the results obtained are discussed.

\section{Electromagnetic Actuator}

The electromagnetic actuator introduces forces that are inversely proportional to the square of the gap. For each coil, the force is given by Eq. (1) [10]. 


$$
F_{E M A}=\frac{N^{2} I^{2} \mu_{0} a f}{2\left((e \pm \delta)+\frac{b+c+d-2 a}{\mu_{r}}\right)^{2}}
$$

The parameters that define the geometry of the coils $\left(a, b, c, d\right.$ e $f$ ) are shown in the Fig. $1 ; \mu_{0}$ and $\mu_{r}$ are the magnetic permeability in the vacuum and the relative permeability of the material, respectively. $\mu_{r}$ is determined experimentally. The gap is given by $e ; \delta$ is the variation of the gap due to the vibration of the rotor at the position of the electromagnetic actuator.

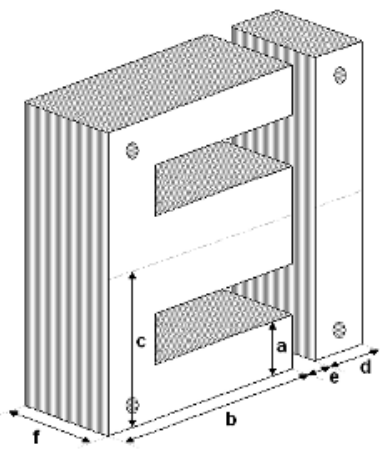

Fig. 1. Ferromagnetic circuit.

The parameters of the coil are described in Tab. (1).

Table 1. Parameters of the coil.

\begin{tabular}{|c|c|}
\hline$\mu_{0}(\mathrm{H} / \mathrm{m})$ & $1.2566 \mathrm{e}-06$ \\
\hline$\mu_{r}$ & 700 \\
\hline$N($ spires $)$ & 278 \\
\hline$a(\mathrm{~mm})$ & 21 \\
\hline$b(\mathrm{~mm})$ & 84 \\
\hline$c(\mathrm{~mm})$ & 63 \\
\hline$d(\mathrm{~mm})$ & 21 \\
\hline$f(\mathrm{~mm})$ & 42 \\
\hline$e(\mathrm{~mm})$ & 0.9 \\
\hline
\end{tabular}

\subsection{Linear Model}

The simple model for the actuator uses the basic equations of electricity and electromagnetim [11], [12] and [13]. So, the linearized actuator is:

$F_{E M A}=K_{i} i-K_{x} x$

where,

$K_{i}=\frac{\partial F_{E M A}}{\partial i}=\frac{N^{2} I_{b} \mu_{0} a f}{2\left(e+\frac{b+c+d-2 a}{\mu_{r}}\right)^{2}}$

$$
K_{x}=-\frac{\partial F_{E M A}}{\partial x}=\frac{N^{2} I_{b}{ }^{2} \mu_{0} a f}{2\left(e+\frac{b+c+d-2 a}{\mu_{r}}\right)^{3}}
$$

where $I_{b}$ is permanent electrical current.

The Fig. 2 presents the electromagnetic force obtained from Eq. (1) (nonlinear model) and Eq. (2) (linear model) with $\delta=0$ ).

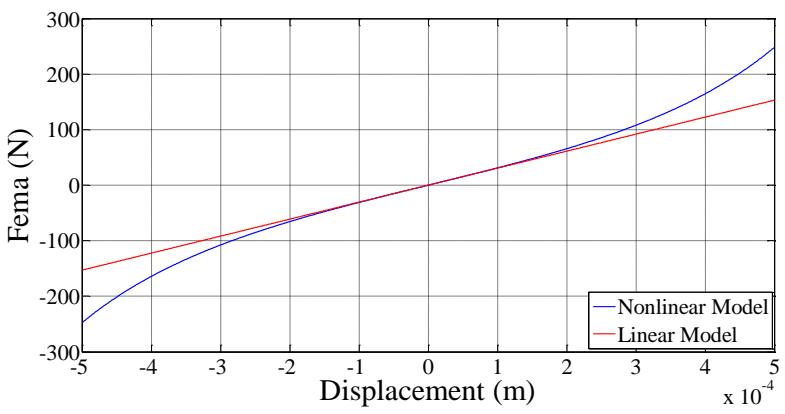

Fig. 2. Comparison between nonlinear and linear models.

Analyzing Fig. 2, it is observed that the electromagnetic force presents a linear behavior for displacements between $-1.5 \times 10^{-4} \mathrm{~m}<x<1.5 \times 10^{-4} \mathrm{~m}$. Usually for this type of applications the rotor displacement $(\delta)$ at the position of the actuator is of the same order of magnitude.

\section{ROTOR MODEL}

The dynamic response of the considered mechanical system can be modeled by using principles of variational mechanics, namely the Hamilton's principle. For this aim, the strain energy of the shaft and the kinetic energies of the shaft and discs are calculated. An extension of Hamilton`s principle makes possible to include the effect of energy dissipation. The parameters of the bearings are included in the model by using the principle of the virtual work. For computation purposes, the finite element method is used to discretize the structure so that the energies calculated are concentrated at the nodal points. Shape functions are used to connect the nodal points. To obtain the stiffness of the shaft the Timoshenko`s beam theory was used and the cross sectional area was updated as proposed by [14]. The model obtained as described above is represented mathematically by the set of differential equations [15] given by Eq. (5).

$$
\begin{aligned}
& {[M]\{\ddot{x}(t)\}+\left[C_{b}+\dot{\phi} C_{g}\right]\{\dot{x}(t)\}+\left[K+\phi K_{g}\right]\{x(t)\}=} \\
& =\left\{F_{u}(t)\right\}+\left\{F_{E M A}(t)\right\}
\end{aligned}
$$

where $\{x(t)\}$ is the vector of generalized displacements; $[M],[K],\left[C_{b}\right],\left[C_{g}\right]$ e $\left[\mathrm{K}_{g}\right]$ are the well-known matrices of inertia, stiffness, bearing viscous damping (that may include proportional damping), Coriolis (with respect to the speed of rotation), and the effect of the variation of the rotation speed; $\dot{\phi}$ is the time-varying angular speed, and $\left\{F_{u}(t)\right\}$ and $\left\{F_{E M A}(t)\right\}$ are the forces due to the 
unbalance and to the electromagnetic actuator, respectively.

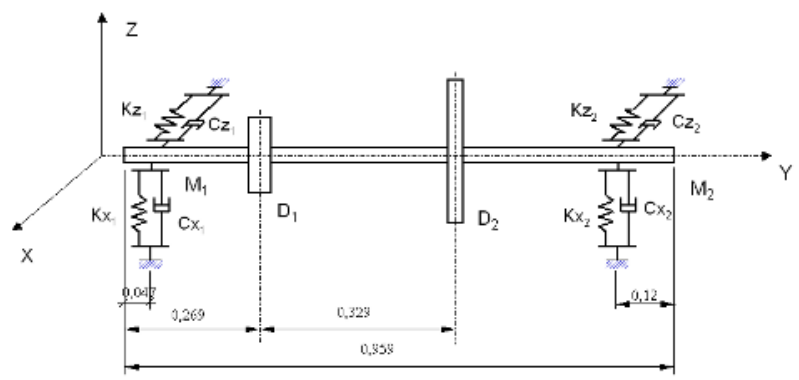

Fig. 3. Rotor geometry.

The finite element model considers 4 d.o.f. per node, namely two displacements (along the directions $x$ and $z$ ) and two rotations (around the axes $x$ and $z$ ), respectively. The model was discretized according to 43 nodes as shown in the Fig. 4 . The ball bearings $\left(B_{1}\right)$ are located at the nodes \#4 and \#5 and the bearing containing the electromagnetic actuator $\left(\mathrm{B}_{2}\right)$ is placed at the node \#39. The first disc $\left(D_{1}\right)$ is placed between the nodes \#12 and \#15; the second disc $\left(D_{2}\right)$ is located between the nodes \#29 and \#31. Finally, concentrated masses were included in the model at the position of the bearings and at the coupling between the shaft and the motor.

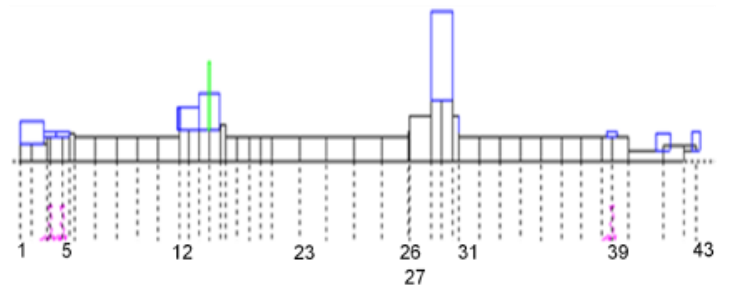

Fig. 4. Rotor Finite Element Model.

The properties used for the shaft are the following: Elastic or Young Modulus $(\mathrm{E})=210 \mathrm{GN} / \mathrm{m}^{2}$, density $=$ $7800 \mathrm{Kg} / \mathrm{m}^{3}$ and Poison Coefficient $=0.3$.

The Tab. (2) shows the physical properties of the rotor.

Table 2. Physical characteristics of rotor.

\begin{tabular}{|c|c|}
\hline \multicolumn{2}{|c|}{ Rotor } \\
\hline Mass of shaft $(\mathrm{kg})$ & 9.690 \\
\hline Mass of disc $\mathrm{D}_{1}(\mathrm{~kg})$ & 2.032 \\
\hline Mass of disc $\mathrm{D}_{2}(\mathrm{~kg})$ & 10.61 \\
\hline Thickness of $\mathrm{D}_{1}(\mathrm{~m})$ & 0.029 \\
\hline Thickness of $\mathrm{D}_{2}(\mathrm{~m})$ & 0.030 \\
\hline Diameter of shaft $(\mathrm{m})$ & 0.040 \\
\hline
\end{tabular}

The Tab. (3) shows the stiffness and damping coefficients of the bearings.
Table 3. Physical characteristics of rotor

\begin{tabular}{|c|c|}
\hline \multicolumn{2}{|c|}{ Bearings } \\
\hline $\mathrm{k}_{\mathrm{x} 1}(\mathrm{~N} / \mathrm{m})$ & $1.168 \times 10^{8}$ \\
\hline $\mathrm{k}_{\mathrm{z} 1}(\mathrm{~N} / \mathrm{m})$ & $1.651 \times 10^{8}$ \\
\hline $\mathrm{k}_{\mathrm{x} 2}(\mathrm{~N} / \mathrm{m})$ & $1.409 \times 10^{6}$ \\
\hline $\mathrm{k}_{\mathrm{z} 2}(\mathrm{~N} / \mathrm{m})$ & $1.434 \times 10^{6}$ \\
\hline $\mathrm{C}_{\mathrm{x} 1}, \mathrm{C}_{\mathrm{x} 2}, \mathrm{C}_{\mathrm{z} 1}, \mathrm{C}_{\mathrm{z} 2}(\mathrm{~N} . \mathrm{s} / \mathrm{m})$ & $280,120,300,120$ \\
\hline
\end{tabular}

\section{LINEAR MATRIX INEQUALITIES (LMIS)}

LMIs have been used in the analysis of dynamical systems for more than 100 years, when Aleksandr Mikhailovich Lyapunov presented his original work, thus introducing the well-known Lyapunov Theory [17]. He demonstrated that the differential equation:

$$
\dot{x}(t)=A x(t)
$$

is stable (all the trajectories converge to zero) if and only if there is a positive-definite matrix $\mathrm{P}$ such that:

$$
A^{T} P+P A>0
$$

The inequality given by Eq. (7) is known as the Lyapunov inequality.

\section{1. $H_{\infty}$ norm}

Boyd showed how to calculate de $H_{\infty}$ norm by using LMIs [17]. The $H_{\infty}$ norm can be solved by using the following optimization convex problem:

$$
\|G\|_{\infty}=\min \mu
$$

subject to $\left[\begin{array}{cc}A^{T} P+P A+C^{T} C & P B \\ B^{T} P & -\mu\end{array}\right]<0$

$$
P>0, \mu>0
$$

where $\mu$ is a scalar.

\subsection{Linear Quadratic Regulator (LQR)}

Several authors have considered applications of LQR, however, not so many discuss the LMI version of this controller [18], [19]. A version of LQR solved by LMIs is found in [20]. The authors of this contribution show that the LQR-LMI problem is described by:

$$
\begin{gathered}
\min _{X, P_{l m i}, X_{l m i}} \operatorname{tr}\left(\left[Q_{l q r}\right]\left[P_{l m i}\right]\right)+\operatorname{tr}\left(\left[X_{l m i}\right]\right)+\operatorname{tr}\left(\left[Y_{l m i}\right] N\right)+\operatorname{tr}\left([N]^{T} Y_{l m i}{ }^{T}\right) \\
\qquad A \rrbracket[P]-[B]\left[Y_{l m i}\right]+[P][A]^{T}-\left[Y_{l m i}\right]^{T}[B]^{T}+\left[B_{w}\right]\left[B_{w}\right]^{T}<0 \\
\text { subject to } \\
{\left[\begin{array}{cc}
{\left[X_{l m i}\right]} & {\left[R_{l q r}\right]^{1 / 2}\left[Y_{l m i}\right]} \\
{\left[Y_{l m i}\right]^{T}\left[R_{l q r}\right]^{1 / 2}} & {\left[P_{l m i}\right]}
\end{array}\right]>0}
\end{gathered}
$$


where $\left[Q_{l q r}\right]$ weights each state, $\left[R_{l q r}\right]$ weights the energy cost of each controller, $N$ is a noise position vector, $\left[X_{l m i}\right]$ and $\left[Y_{l m i}\right]$ are the LMIs solutions, and $\operatorname{tr}()$ denote the matrix trace.

\section{FUZZY LOGIC CONTROL (FLC)}

Der Hagopian et al. used fuzzy logic approach to control light structures [8] as shown in Fig. 5.

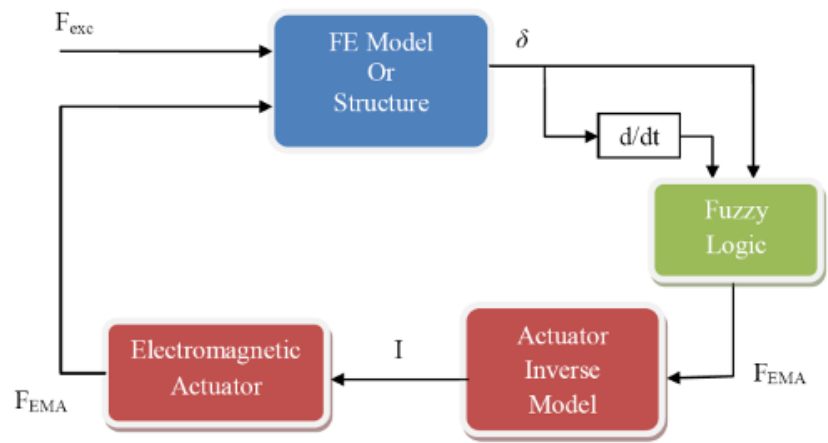

Fig. 5. Control strategy.

The first step in this approach consists in the fuzzification of the physical inputs, which are converted into several fuzzy variables. The number of fuzzy variables depends on the number of membership functions used.

In this work two membership GBELL Matlab ${ }^{@}$ functions "Positive" and "Negative" are used and presented in Fig. 6.

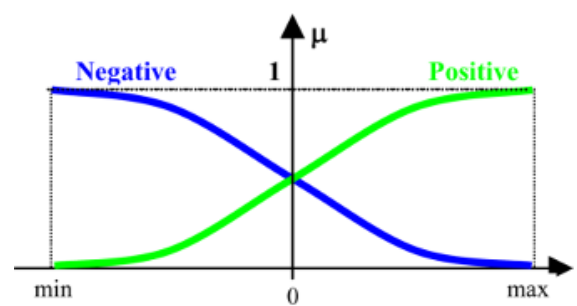

Fig. 6. Input membership functions [8].

The inference engine implements the "minimum" function $w_{j}$. The rules are presented in Tab. 4 .

Table 4. Fuzzy controller rules.

\begin{tabular}{|c|c|c|}
\hline Rule & Condition & Decision \\
\hline 1 & $\begin{array}{l}\text { IF positive displacement AND positive } \\
\text { velocity }\end{array}$ & Action \\
\hline 2 & $\begin{array}{l}\text { IF positive displacement AND negative } \\
\text { velocity }\end{array}$ & $\begin{array}{l}\text { No } \\
\text { action }\end{array}$ \\
\hline 3 & $\begin{array}{l}\text { IF negative displacement AND positive } \\
\text { velocity }\end{array}$ & $\begin{array}{l}\text { No } \\
\text { action }\end{array}$ \\
\hline 4 & $\begin{array}{c}\text { IF positive negative AND negative } \\
\text { velocity }\end{array}$ & Action \\
\hline
\end{tabular}

Finally the command is obtained after defuzzification. This defuzzification requires the knowledge of the fuzzy output variables corresponding to the rules and the aggregation of these rules as well as the output membership functions. In this paper the Takagi-Sugeno method is used for its well adaptation to the controller real time computation. The membership functions are the following:

$$
z_{1}=0 \text { and } z_{2}=100 \delta+1000 \dot{\delta}
$$

The command force $F$ (Fig. 7) is given by the output of the controller and can be written as:

$$
F=\frac{\sum_{j=1}^{2} w_{j} z_{j}}{\sum_{j=1}^{2} w_{j}}
$$

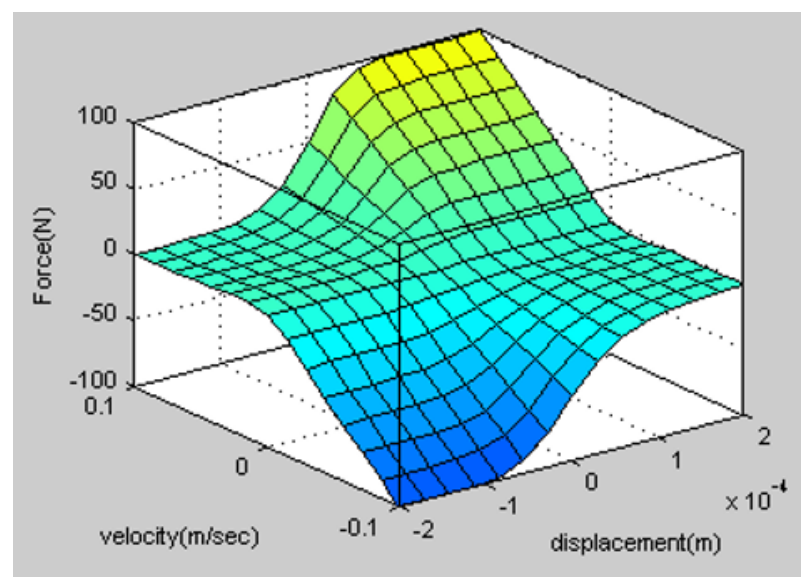

Fig. 7. Fuzzy controller surface.

\section{RESULTS AND DISCUSSION}

This section presents the results obtained, using the linear and the nonlinear actuator models. In the linear model, the gain of the controller is determined by using $H_{\infty}$ norm and LQR, both solved by LMIs.

In the nonlinear case, the fuzzy logic was used to determine the control force. In this situation the inverse model of actuator is used, and the electrical current is determined by:

$I=\sqrt{\frac{2 F_{E M A}\left((e \pm \delta)+\frac{b+c+d-2 a}{\mu_{r}}\right)^{2}}{N^{2} \mu_{0} a f}}$

Three control cases were analyzed: impulsive input, run-up test (from 0 to $5000 \mathrm{rpm}$ ) and run-down test (from 5000 to $0 \mathrm{rpm}$ ). In both cases, the excitation force was applied at the disk \#2 (see Fig. 3).

In the impulsive case, the system response was analyzed during $1 \mathrm{sec}$, while for run-up and for run-down cases, it was analyzed for $100 \mathrm{sec}$. 


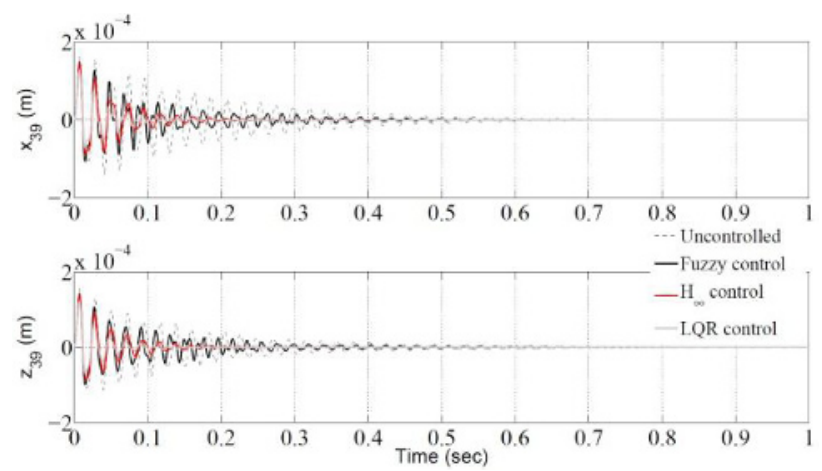

Fig. 8. Controlled and uncontrolled impulse responses.

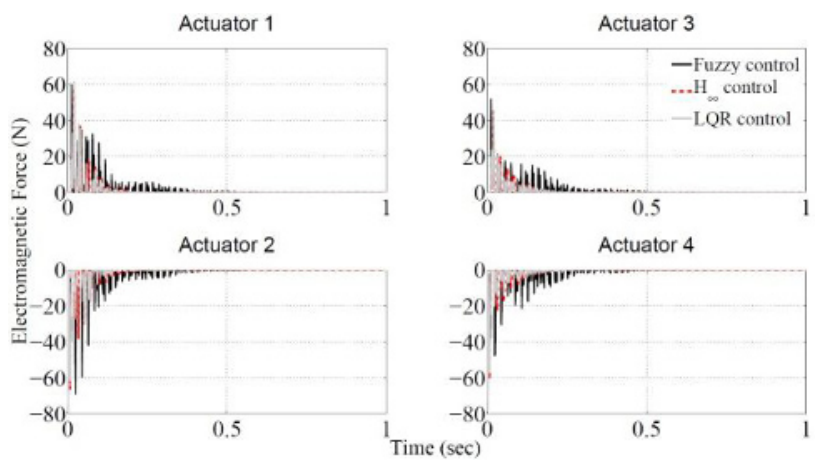

Fig. 9. Electromagnetic force for the impulse test.

Both the controllers ( $H_{\infty}$ norm and LQR) were efficient in the impulse test and presented similar behavior as shown in the Fig. 8. The best results occurred for the linear case (both in terms of the rotor response and the electromagnetic force). Fig. 9 presented the electromagnetic forces for the four actuators. It is important to observe that the actuator applies an attraction force on the system so that actuators 2 and 4 present negative force values.

Following, the obtained results for run-up and rundown are presented.
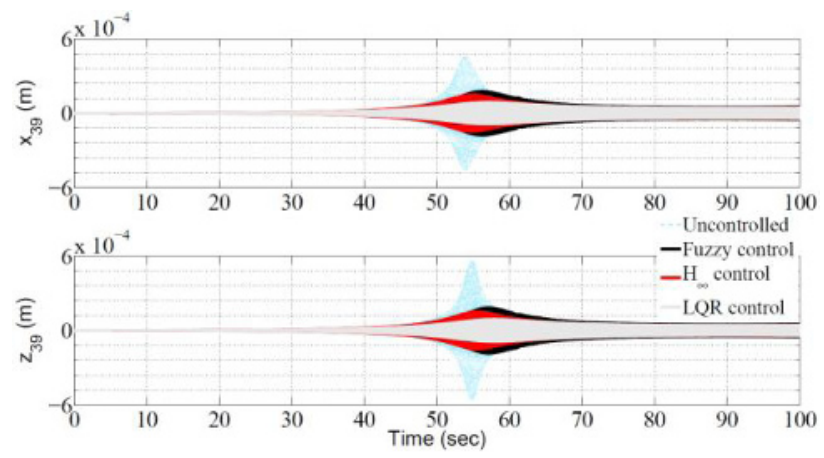

Fig. 10. Controlled and uncontrolled system: run-up test.

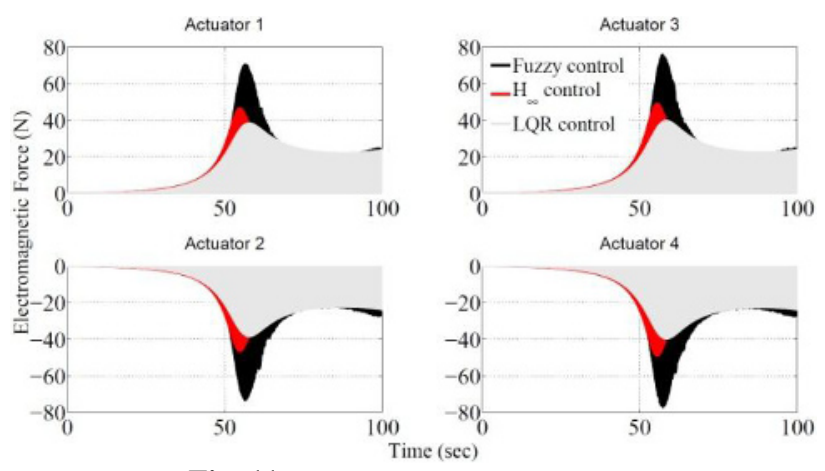

Fig. 11. Electrical current: run-up test.
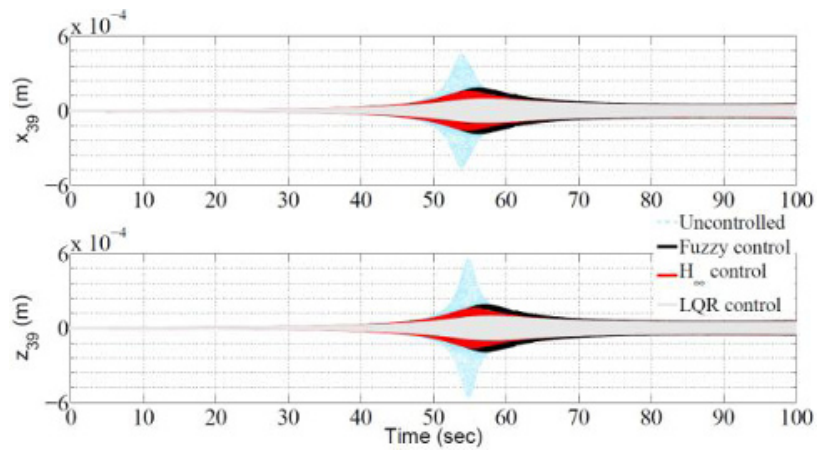

Fig. 12. Controlled and uncontrolled system: run-down test.

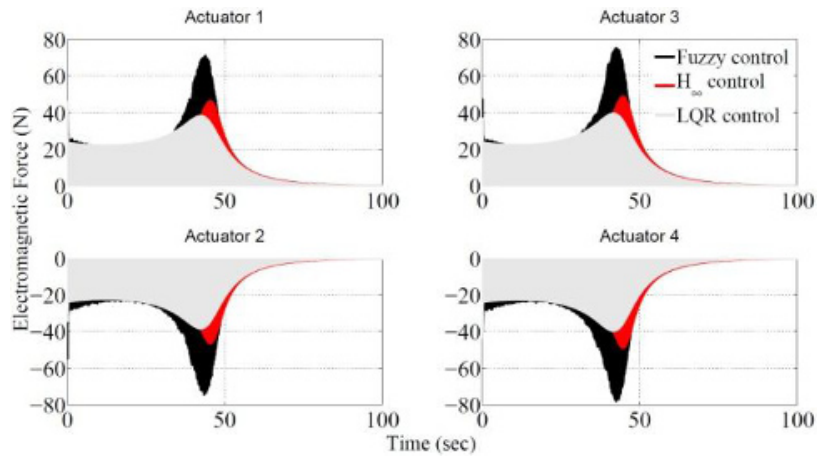

Fig. 13. Electrical current: run-down test.

Figs. (10) to (13) demonstrate that the actuator is able to control the dynamic behavior of the rotor both in the acceleration and deceleration tests. It is important to notice that the linear displacement range was mostly respected in the LQR control. The optimal control showed better results than $H_{\infty}$ norm. It is possible to notice that the LQR control led to larger reduction in the rotor displacement.

In terms of the fuzzy logic technique, it is possible to notice again that the system was controlled as expected. The behavior is similar to the one obtained by using $H_{\infty}$ norm, in terms of displacement responses (see Fig. 10 and 12). However, this type of controller requires larger electromagnetic force as compared with the linear case.

One of the possible causes for the difference found in the values of the electromagnetic force is due to the presence of nonlinearities in the fuzzy logic controller.

It is worth mentioning that the fuzzy controller should be a good alternative to handle uncertainties that are commonly present in the context of experimentation. 


\section{CONCLUSIONS}

In the present contribution the results obtained with a nonlinear actuator model were compared to those from the linear model. In the linear case, the controllers were designed for $H_{\infty}$ norm and LQR, both solved by LMIs.

The presented results showed that the fuzzy logic approach permits to take into account the nonlinearity of the actuator. Moreover, the controlled response is comparable to the one found for the linearized electromagnetic actuator.

The linearization of the actuator made possible the determination of the controller gains. It is important to emphasize that comparison is only possible in the linear range since only the fuzzy controller is valid outside this range.

In the linear case, both as the $H_{\infty}$ norm and LQR were solved by LMIs. The choice of this tool is due to the possibility of including uncertainties in the system and its simplicity of implementation.

\section{ACKNOWLEDGEMENTS}

The authors are thankful to the Brazilian Research Agencies FAPEMIG, CNPq and CAPES for the financial support to this work through the INCT-EIE.

\section{References}

1. BUENO, D. D, Active Vibration Control and Optimal Placement of Piezoelectric Sensors and Actuators. Master of Science in Mechanical Engineering - Faculdade de Engenharia, Universidade Estadual Paulista, Ilha Solteira, Brazil (2007).

2. C. R. Fuller, S. J. Elliot, P. A. Nelson, Active Control of Vibration, Academic Press (1996).

3. C. H. Hansen, S. D. Snyder, Active Control of Noise and Vibrations, E\&FN Spon, London UK (1997).

4. W. Gawronski, Dynamics and Control of Structures: A Modal Approach, 1.ed. New York: Springer Verlag, 231p, (1998).

5. J. Juang, Q. Phan, Identification and Control of Mechanical System, Cambridge University Press, ISBN 0521783550 (2001).

6. F. Liu, L. Zhang, Modal-Space Control of Flexible Intelligent Truss Structures via Modal Filters, Proceeding of IMAC - International Modal Analysis Conference, p. 187-193 (2000).

7. E. H. Koroishi, E. A. Perini, L. P. Nasicmento, V. Lopes Jr, V. Steffen Jr, Modal Active Vibration Control in Rotor System Using Magnetic Bearings with $\mathrm{H}_{\infty}$ norm, Proceedings of the 1rst International Congress of Mathematics, Engineering and Society ICMES 2009 (2009).

8. J. Der Hagopian, J. Mahfoud, Electromagnetic actuator design for the control of light structures, Smart Structures and Systems, Vol. 6, No. 1, 29-38 (2010).
9. M. Mahlis, L. Gaudiller, J. Der Hagopian, Fuzzy modal active control of the dynamic behavior of flexible structures, J. Vib. Control, 11, 67-88 (2005).

10. G. Damien, Circuit Magnétique - Électroaimant, eLEE, e-learning for Electrical Engineering, http://www.lei.ucl.be/multimedia/eLEE/FR/realisatio ns/CircuitsElectriques/index.htm (2003).

11. E. Maslen, Magnetic Bearings, University of Virginia, Department of Mechanical, Aerospace and Nuclear Engineering, Charlottesville, Virginia, 233 p (2000).

12. R. M. Furtado, A Magnetic Actuator Development for Contactless Excitation in Rotor Systems, Campinas, Faculdade de Engenharia Mecânica, Universidade Estadual de Campinas, 113p (2008).

13. E. A. Perini, Redução de Vibrações em Rotores Utilizando Atuadores Magnéticos e Sistema de Controle Feedforward, Dissertação de Mestrado, Faculdade de Engenharia - Campus de Ilha Solteira, Universidade Estadual Paulista, Ilha Solteira, 2009.

14. J. H. Hutchison, Shear Coefficients for Timoshenko Beam Theory, Journal of Applied Mechanics, January (2001).

15. M. Lalanne, G. Ferraris, Rotordynamics Prediction in Engineering, 2nd edition, Jhon Wiley and Sons, New York (1997).

16. R. C. Simões, Optimal Modal Control of a Flexible Rotor Using Piezoelectric Stack Actuators, $133 \mathrm{f} . \mathrm{Ph}$. d. Thesis, Federal University of Uberlândia, Uberlândia (2006).

17. S. Boyd, V. Balakrishnan, E. Feron, L. El Ghaoui, Linear Matrix Inequalities in Systems and Control Theory, Siam Studies in Applied Mathematics, USA, 193p (1994).

18. V. Balakrishnan, Linear Matrix Inequalities in Robust Control a Brief Survey, School of Electrical and Computer Engineering, Purdue University, West Lafayette, IN 47907-1285, USA.

19. E. A. Johnson, B. Erkus, Structural Control with Dissipative Camping Devices, American Control Conference, ACC, Anchorage, Alaska (2002).

20. B. Erkus, Y. J. Lee, Linear Matrix Inequalities and Matlab LMI Toolbox, University of Southern California Group Meeting Report, Los Angeles, California (2004). 